Depinning field of a periodic domain wall array in vicinal nanowires

Ana L. Dantas, G. O. G. Rebouças, and A. S. Carriço

Citation: Journal of Applied Physics 105, 07 C116 (2009);

View online: https://doi.org/10.1063/1.3067476

View Table of Contents: http://aip.scitation.org/toc/jap/105/7

Published by the American Institute of Physics

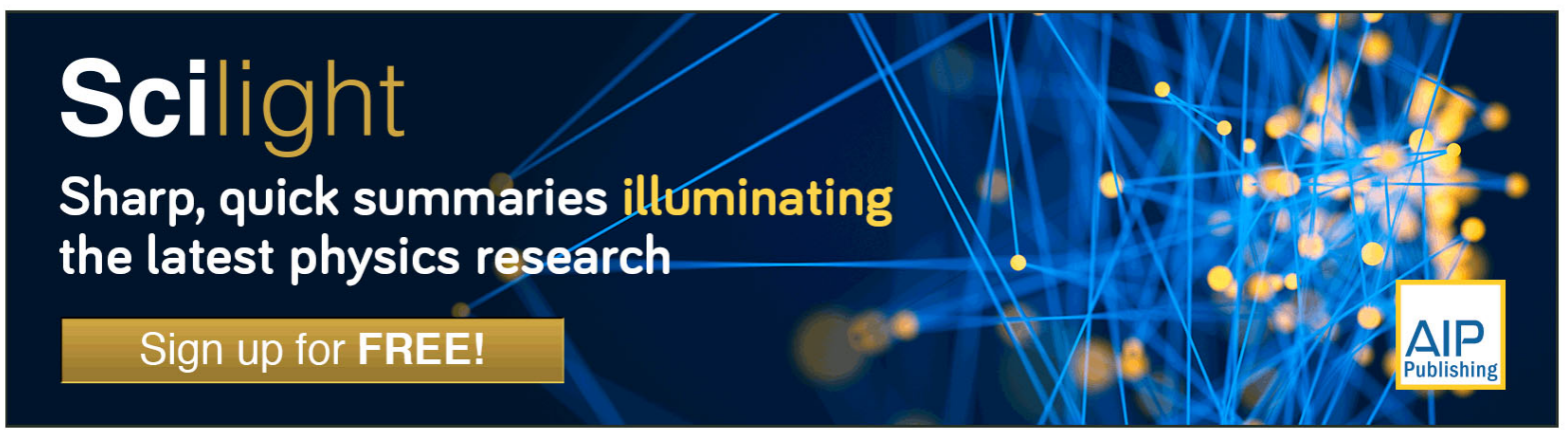




\title{
Depinning field of a periodic domain wall array in vicinal nanowires
}

\author{
Ana L. Dantas, ${ }^{1}$ G. O. G. Rebouças, ${ }^{2}$ and A. S. Carriço, ${ }^{2, a)}$ \\ ${ }^{1}$ Departamento de Física, Universidade do Estado do Rio Grande do Norte, 59610-210 Mossoró, \\ Rio Grande do Norte, Brazil \\ ${ }^{2}$ Departamento de Física, Universidade Federal do Rio Grande do Norte, 59078-970 Natal, \\ Rio Grande do Norte, Brazil
}

(Presented 13 November 2008; received 5 October 2008; accepted 2 November 2008; published online 11 March 2009)

\begin{abstract}
We report a theoretical investigation of the magnetic states and depinning field of a periodic array of head-to-head domain walls of flat Fe rectangular nanowires, exchange coupled with a vicinal two-sublattice uniaxial antiferromagnetic substrate. We show that for strong interface exchange energy, domain walls are pinned at interface steps perpendicular to the antiferromagnetic easy axis, separating terraces with opposite interface exchange field. The array sequence, which alternates head-to-head and tail-to-tail domain walls, may form a structure with alternate chirality or with the same chirality. The domain wall dipolar field affects the chirality sequence, which is tunable by the geometrical constraints and the strength of the interface exchange field. The depinning field of 10 $\mathrm{nm}$ thick, $1 \mu \mathrm{m}$ long wires, with widths of 100 and $200 \mathrm{~nm}$, is of the order of the interface field strength, and the depinning process involves domain wall motion and the transversal displacement of a periodic array of vortices. (C) 2009 American Institute of Physics. [DOI: 10.1063/1.3067476]
\end{abstract}

The recent interest in the study of domain walls in constrained geometries is largely motivated by the promise of a high performance nonvolatile memory nanodevice, with data storage on trains of 10-100 head-to-head domain walls in ferromagnetic (F) nanowires. ${ }^{1,2}$ The domain walls of $1 \mu \mathrm{m}$ long flat wires, with transverse dimensions on the order of a few hundred nanometers, behave as quasiparticles which can be manipulated by external magnetic fields and spinpolarized currents. Variations in the wire geometry, by introducing constrictions and protrusions, is currently being investigated as a means of modifying the domain wall structure and setting a pinning barrier to domain wall motion. ${ }^{3}$

Exchange coupling the $\mathrm{F}$ nanowire to a vicinal antiferromagnetic (AF) substrate is another promising way of controlling the domain wall structure, the pinning barrier to domain wall motion, and the depinning mechanisms. Vicinal AF substrates may be chosen to have the F/AF interface with a periodic sequence of terraces composed of spins of opposite AF sublattices, separated by steps perpendicular to the direction of the AF spins. ${ }^{4,5}$ The periodic change in the interface exchange energy leads to the nucleation of interface pinned walls at the step edges. The interface exchange pinning energy wells are the counterpart of the geometrical constrictions in race-track memories and control the motion of the walls along the stripe.

The nucleation of domain walls in the F nanowire, at the edges of the interface steps, separating domains in a one-toone correspondence to the interface exchange energy pattern, requires an interface energy threshold which compensates for the cost of the intrinsic exchange and dipolar energies. For a given pair of $\mathrm{F}$ and $\mathrm{AF}$ materials this can be accomplished by choosing the miscut angle small, so that the terrace length is

\footnotetext{
a) Author to whom correspondence should be addressed. FAX:+55-8432153791. Electronic mail: acarrico@dfte.ufrn.br.
}

large enough to meet the required interface energy per domain. ${ }^{5}$ The terrace length and the stripe width both affect the threshold value of the interface field. The threshold value of the interface field strength for a $1.6 \mu \mathrm{m}$ long and $100 \mathrm{~nm}$ wide Fe stripe with terraces of $200 \mathrm{~nm}$ is $0.3 \mathrm{kOe}$, and the wall width is around $120 \mathrm{~nm}$. Transverse walls do not form for smaller values of the interface field strength. Instead one finds asymmetric walls with a single vortex at one side of the wall center.

In this paper we present a discussion of the magnetic states and depinning processes of an array of domain walls of a flat rectangular iron wire exchange coupled to a vicinal AF substrate. We show that the depinning of the domain walls is tunable by the interface exchange energy, the wire width, and the terrace length. We also show that the dipolar interaction of neighboring domain walls affects the chirality sequence. Large values of wire width favor the formation of wide walls in which the stray field may cover a considerable fraction of the terrace length, leading to the nucleation of walls with alternate chirality (AC).

We consider $1.6 \mu \mathrm{m}$ long and $10 \mathrm{~nm}$ thick rectangular iron wires with widths of 100 and $200 \mathrm{~nm}$ exchange coupled to a vicinal two-sublattice AF substrate. The AF substrate spins are oriented along the wire longitudinal direction $(x$ axis), so that the interface exchange field favors the formation of an array of head-to-head domain walls, as shown in Fig. 1.

The AF substrate is held frozen in the AF order, and the interface exchange coupling is represented by an effective field. The wire magnetic structure is described in terms of the magnetic moments of a monolayer of cubic cells with edge $d$, and the energy density is written as a sum of intrinsic exchange energy, interface exchange energy, Zeeman and anisotropy energies, and dipolar energy: 


$$
E=\frac{A}{d^{2}} \sum_{j} \sum_{k}\left(1-\hat{m}_{j} \cdot \hat{m}_{k}\right)-M_{S} \vec{H}_{I} \cdot \sum_{i} \hat{m}_{i}-M_{S} \vec{H} \cdot \sum_{j} \hat{m}_{j}-K \sum_{j}\left(m_{j}^{x}\right)^{2}+\frac{M_{S}^{2}}{2} \sum_{j} \sum_{k}\left(\frac{\hat{m}_{j} \cdot \hat{m}_{k}}{n_{j k}^{3}}-\frac{3\left(\hat{m}_{j} \cdot \hat{n}_{j k}\right)\left(\hat{m}_{k} \cdot \hat{n}_{j k}\right)}{n_{j k}^{5}}\right),
$$

where $A$ is the $\mathrm{F}$ exchange stiffness, $M_{S}$ is the saturation magnetization, $\hat{m}_{i}$ is the direction of the magnetic moment of the $i$ th cell, and $n_{i j}$ is the distance between cells $i$ and $j$ in units of cell size. We use $d=10 \mathrm{~nm}$ and the following values for the iron parameters: exchange stiffness $A=2.5$ $\times 10^{-11} \mathrm{~J} / \mathrm{m}$, anisotropy constant $K=4.7 \times 10^{4} \mathrm{~J} / \mathrm{m}^{3}$, and saturation magnetization $M_{S}=1.7 \times 10^{6} \mathrm{~A} / \mathrm{m}^{6-8}$ The equilibrium states are found using a self-consistent local field algorithm, which allows calculating the orientation of the magnetic moment in each cell. ${ }^{9-12}$

For each value of the external field, the self-consistent procedure is initialized with the magnetic state corresponding to the equilibrium state of the previous value of the external field. Proceeding this way we find the metastable equilibrium state nearest to the preceding one, as appropriate to modeling the depinning of the array of domain walls from the pinning centers located at the step edges.

There are two special ways of arranging domain walls in a vicinal wire with finite terrace length $\left(L_{x}\right)$. If the terrace length is within the reach of the dipolar field of a domain wall, then neighboring walls will arrange according to the dipolar interaction. For narrow wires $\left(L_{y}=100 \mathrm{~nm}\right)$ the dipolar interaction favors the nucleation of an array of walls with the same chirality (SC). Arrays with neighboring walls with AC are favored for larger values of the wire width $\left(L_{y}\right.$ $=200 \mathrm{~nm}$ ).

The leading features of the stray field may be seen from Fig. 1. In the right column panel we display the map of the dipolar field of a wall nucleated at a single step of a $1.6 \mu \mathrm{m}$ long wire with $L_{y}=200 \mathrm{~nm}$. The wall has reflection symmetry with respect to its center and the domain wall width is not uniform. The wall is $\mathrm{V}$ shaped (as previously reported ${ }^{13,14}$ ), being wider $(100 \mathrm{~nm})$ at the upper edge of the wire and narrower $(40 \mathrm{~nm})$ at the lower edge. The curves in the right column panel show the values of $H_{d}^{y}$ as a function of the distance to the wall center for lines running across the domain wall. As seen in the white color curve, for $y=200 \mathrm{~nm}$,

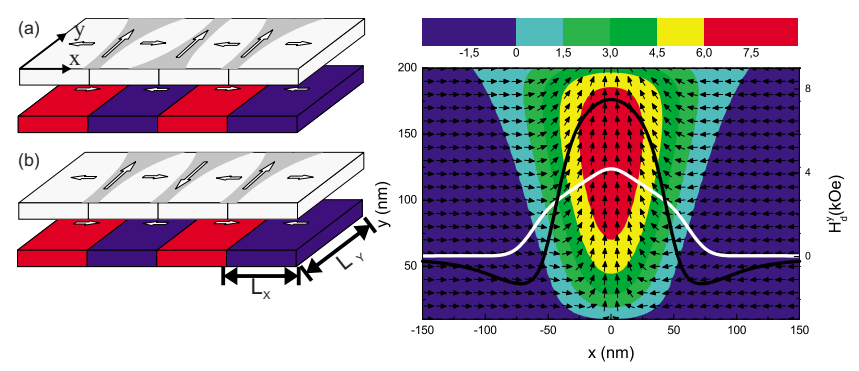

FIG. 1. (Color online) Schematic of (a) AC and (b) SC arrays of head-tohead domain walls of vicinal nanowires. The right hand side panel shows the dipolar field of a domain wall nucleated at a single step defect of a 10 $\mathrm{nm}$ thick and $1.6 \mu \mathrm{m}$ long iron wire of width $L_{y}=200 \mathrm{~nm}$ for an interface field strength of $1.47 \mathrm{kOe}$. The color bar code indicates the value of the transverse component $\left(H_{d}^{y}\right)$ of the dipolar field.
$H_{d}^{y}$ is always positive at the upper edge of the wire, reaching $4 \mathrm{kOe}$ at the wall center. In the middle of the wire, as seen in the black curve for $y=100 \mathrm{~nm}, H_{d}^{y}$ reaches the maximum value $(8 \mathrm{kOe})$ at the center and decays to zero at a $50 \mathrm{~nm}$ distance from the wall center. Roughly speaking, the $y$ component of $H_{d}^{y}$ is predominantly positive around the wall center, leading to the formation of neighboring walls with positive $y$ component of magnetization. In the domain wall array, neighboring walls adapt mutually to the dipolar field of each other and the AC sequence is favored for $L_{y}=200 \mathrm{~nm}$. For narrow wires $\left(L_{y}=100 \mathrm{~nm}\right)$ the tails with negative value of $H_{d}^{y}$ is the prevailing factor and favors the nucleation of neighboring walls with the SC.

Another interesting feature of the dipolar effect, as shown schematically in Fig. 1 and in the spin maps of Figs. 3 and 4, is that the $\mathrm{AC}$ sequences are composed of $\mathrm{V}$-shaped walls arranged in a V-up/V-down sequence. The SC sequences composed of $\mathrm{V}$-shaped walls are arranged in the same manner. In the AC sequences the dipolar field emanating from a V-up wall is larger at the upper edge of the wire and leads to a dipolar compression of neighboring walls in the upper edge. In the SC sequences the dipolar field of a V-up wall is more important at the lower edge, leading neighboring walls to compressed in the same manner.

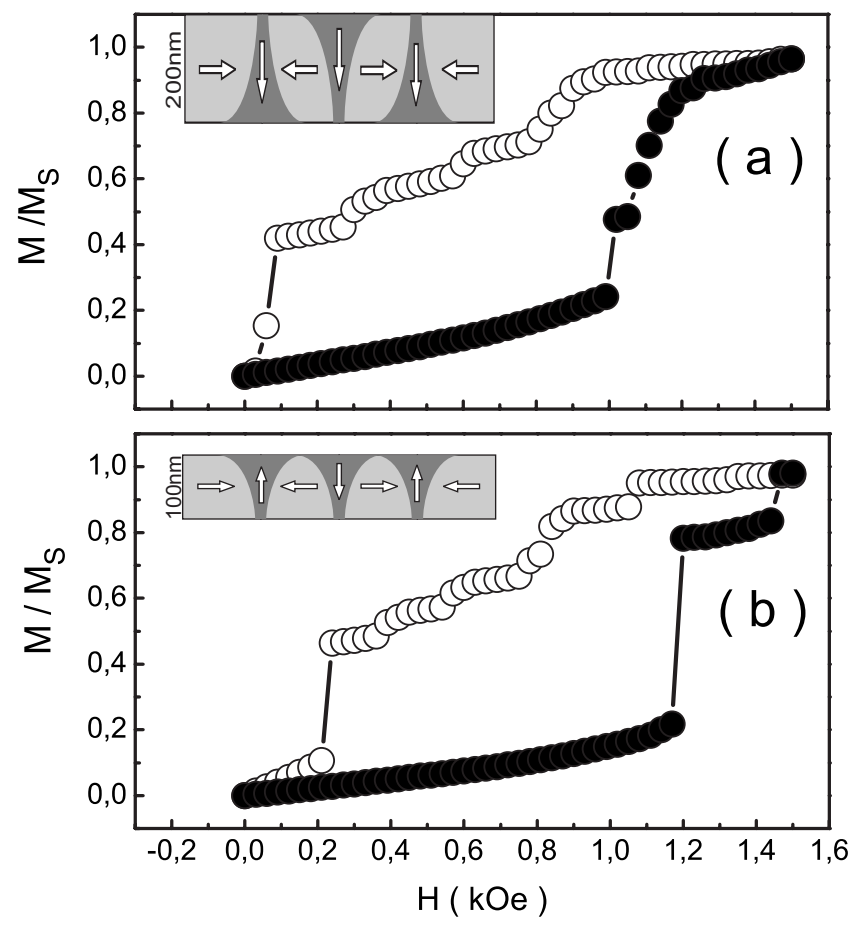

FIG. 2. Depinning curves for $1.6 \mu \mathrm{m}$ long iron wires with $L_{x}=200 \mathrm{~nm}$ terraces (full symbol curves) and $L_{x}=100 \mathrm{~nm}$ (open symbol curves), for (a) an AC sequence with $L_{y}=200 \mathrm{~nm}$ and (b) a SC sequence with $L_{y}$ $=100 \mathrm{~nm}$. In the inset we show schematically the arrangement of V-shaped walls for each case. 

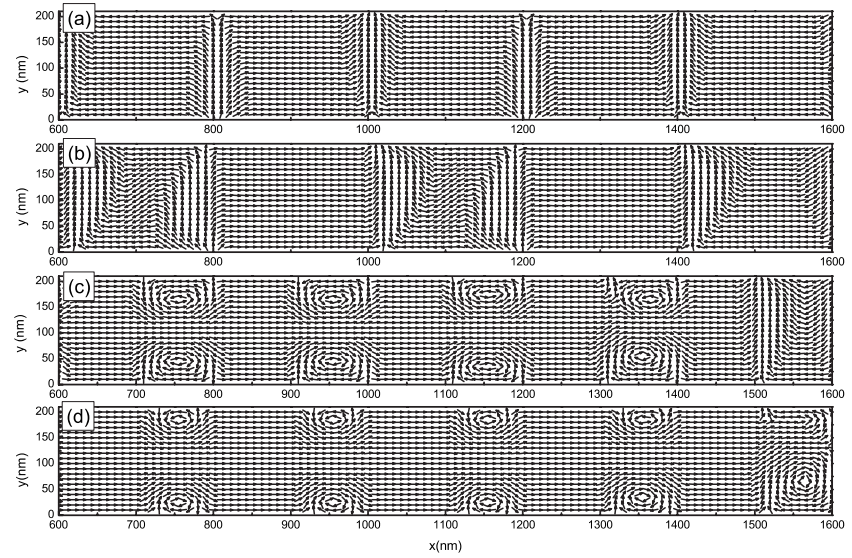

FIG. 3. Spin profiles for selected flat wire geometries showing (a) AC, $200 \mathrm{~nm} \times 200 \mathrm{~nm}^{2}, H=0$; (b) AC, $200 \mathrm{~nm} \times 200 \mathrm{~nm}^{2}, H=0.99 \mathrm{kOe}$; (c) AC, $100 \mathrm{~nm} \times 200 \mathrm{~nm}^{2}, H=0.25 \mathrm{kOe}$; and (d) AC, $100 \mathrm{~nm} \times 100 \mathrm{~nm}^{2}$, $H=0.78 \mathrm{kOe}$.

In Fig. 2(a) we show the magnetization curves corresponding to the depinning process of $10 \mathrm{~nm}$ thick and $1.6 \mu \mathrm{m}$ long wires for AC sequences and $L_{y}=200 \mathrm{~nm}$. The lower curve, for $L_{x}=200 \mathrm{~nm}$, shows a gradual increase in the magnetization corresponding to a gradual compression of the domains opposite to the external field, as shown in Figs. 3(a) and $3(\mathrm{~b})$, where we show the spin maps of the last $1 \mu \mathrm{m}$ of the wire. For $L_{x}=100 \mathrm{~nm}$, the depinning process starts at a smaller value of $H$, with the nucleation of pair of vortices at the domains opposite to the external field, which moves toward the wire edges as $H$ increases, as shown in Figs. 3(c) and $3(\mathrm{~d})$.

The depinning process of an $L_{y}=100 \mathrm{~nm}$ wire, shown in Fig. 2(b), has similar features. For $L_{x}=200 \mathrm{~nm}$ there is a gradual increase in the magnetization, with the motion, in opposite directions, of neighboring walls, leading to a compression of the domain opposite to the external field, as shown in the spin maps of Figs. 4(a) and 4(b). For $L_{x}$ $=100 \mathrm{~nm}$, the depinning process corresponds to the nucleation and motion toward the lower wire edge of vortices at the domains opposite to the external field, as shown in Figs. 4(c) and 4(d). Notice that in all cases the final depinning occurs at an external field strength of the order of the interface field value.

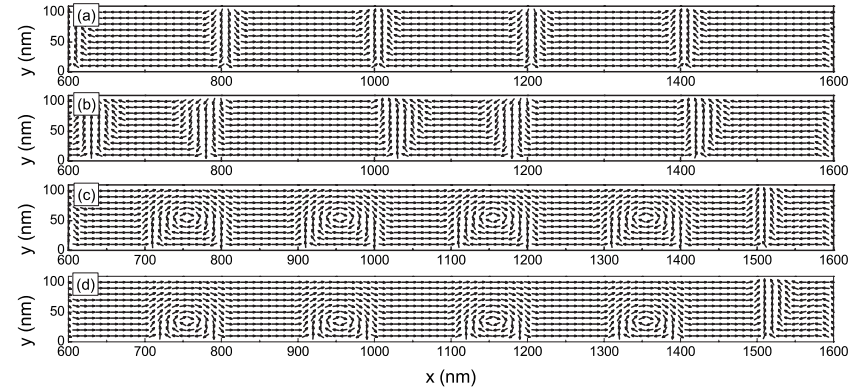

FIG. 4. Spin profiles for selected flat wire geometries showing (a) SC, $200 \mathrm{~nm} \times 100 \mathrm{~nm}^{2}, H=0$; (b) SC, $200 \mathrm{~nm} \times 100 \mathrm{~nm}^{2}, H=1.17 \mathrm{kOe}$; (c) $\mathrm{SC}, 100 \mathrm{~nm} \times 100 \mathrm{~nm}^{2}, H=0.24 \mathrm{kOe}$; and (d) SC, $100 \mathrm{~nm} \times 100 \mathrm{~nm}^{2}$, $H=0.72 \mathrm{kOe}$.

We notice that the effects of the geometrical constrictions on the structure of the domain walls depend on the balance between the dipolar field and the exchange stiffness. We are currently investigating vicinal bilayers with Permalloy and Co stripes. ${ }^{15}$

The authors acknowledge financial support from CAPES and FAPERN. The work of A.S.C. was supported by CNPq Grant No. 350773 and the work of A.L.D. was supported by CNPq Grant No. 309676.

${ }^{1}$ S. S. P. Parkin, M. Hayashi, and L. Thomas, Science 320, 190 (2008).

${ }^{2}$ M. Klaui, J. Phys.: Condens. Matter 20, 313001 (2008).

${ }^{3}$ P. O. Jubert, R. Allenspach, and A. Bischof, Phys. Rev. B 69, 220410(R) (2004).

${ }^{4}$ J. Unguris, R. J. Celotta, and D. T. Pierce, Phys. Rev. Lett. 67, 140 (1991).

${ }^{5}$ E. J. Escorcia-Aparicio, H. J. Choi, W. L. Ling, R. K. Kawakami, and Z. Q. Qiu, Phys. Rev. Lett. 81, 2144 (1998).

${ }^{6}$ F. Porrati and M. Huth, J. Appl. Phys. 85, 3157 (2004).

${ }^{7}$ M. Grimsditch, G. K. Leaf, H. G. Kaper, D. A. Karpeev, and R. E. Camley, Phys. Rev. B 69, 174428 (2004).

${ }^{8}$ C. A. F. Vaz, J. A. C. Bland, and G. Lauhoff, Rep. Prog. Phys. 71, 056501 (2008).

${ }^{9}$ R. E. Camley, Phys. Rev. B 35, 3608 (1987).

${ }^{10}$ A. S. Carriço and R. E. Camley, Phys. Rev. B 45, 13117 (1992).

${ }^{11}$ A. L. Dantas and A. S. Carriço, Phys. Rev. B 59, 1223 (1999).

${ }^{12}$ A. L. Dantas, G. O. G. Rebouças, A. S. W. T. Silva and A. S. Carriço, J. Appl. Phys. 97, 10K105 (2005)

${ }^{13}$ R. D. McMichael and M. J. Donahue, IEEE Trans. Magn. 33, 4167 (1997).

${ }^{14}$ Y. Nakatani, A. Thiaville, and J. Miltat, J. Magn. Magn. Mater. 290-291, 750 (2005)

${ }^{15}$ A. L. Dantas, G. O. G. Rebouças, and A. S. Carriço (to be published). 